\title{
Manufacturing and Application of Numerical Control Equipment for Template of Nano-Carbon Granule Superhydrophobic Deposition
}

\author{
Guangrui Shang, Yan Li \\ Jilin Teachers Institute of Engineering and Technology \\ China Changchun 130052
}

Keywords: Numerical Control, Template, Uniformity, Superhydrophobicy.

\begin{abstract}
The preparation of superhydrophobic template using flame which contains Nano-carbon particles is one of the methods of surface modification. However it is difficult to keep the uniformity of the template. In this paper, the superhydrophobic template device of NC with Nano-carbon particles layer was designed and produced. It has been shown by SEM imagines that the deposition layer on the substrate of glass and stainless steel consisting Nano-carbon particles by burning kerosene, soybean oil and candles respectively is uniformity and the static contact angles is more than $150^{\circ}$. It gives a novel idea for fabricating superhydrophobic template.
\end{abstract}

\section{Introduction}

Material surface modification has gained much attention in recent years. One of the ideas is to design and mimic self-cleaning surfaces ${ }^{[1-3]}$. Many biology surfaces in nature are superhydrophobic, such as the surface of the lotus leaf and butterfly wings ${ }^{[4,5]}$. Mimicking its surface morphology has resulted in a considerable amount of artificial materials ${ }^{[6,7]}$ and developed many applications in industrial and biological fields ${ }^{[8 \sim 10]}$. Micron-scale air "clusters" are trapped in the depressions of the rough surface beneath the water droplets ${ }^{[11 \sim 12]}$. This compound interface results in the increase of micro-contact angle and the decrease of the contact angle hysteresis, which makes the rolling of the water droplets easier and can take the pollutants away from the surface. However, organic liquids such as alcohols or oils can significantly reduce their own surface tension and induce even surface wetting. The droplet will change state from the hole above the concave (Cassie - Baxter state) to fully wet (Wenzel state) ${ }^{[11]}$. No natural surface can show that it is greater than $150^{\circ}$ of the static contact angle and below $10^{\circ}$ of the rolling angle. Such surface is superhydrophobic ${ }^{[13]}$.

In recent years, there have been some literature reports which have been reported that using the candle soot as the medium to prepare the nanometer coating on metal or non-metal substrate $^{[14]}$.However, the uniformity of the layer must to be solved in the process. It is difficult to keep homogeneity. In this work, the numerical control equipment has been assembled. It keeps the coating uniformity. A stepping motor drives the mechanical gearbox and the speed reduction gear for transmission output speed (Shown in Fig.1). The superhydrophobic template consisting of Nano-carbon particles has been made by depositing granules above on the substrate such as glass or stainless steel. Nano-carbon particles are made of the burn of kerosene, soybean oil and candle. It laid the foundation for the further preparation of superhydrophobic surface.

\section{Basic parameters of numerical control device}

The numerical control device guarantees the precise motion of $\mathrm{X}, \mathrm{Y}$ and $\mathrm{Z}$ direction. In $\mathrm{X}$ direction, $v_{x}=3 \sim 60(\mathrm{~mm} / \mathrm{min}), S_{x}=0 \sim 300 \mathrm{~mm}$. It keeps the accuracy is for 5 micrometer.

The motion of $Z$ direction is to use adjusting the height of flame in order to test the scale of out flame, flame core and there distribution. In the process of test, the substrate is pure cope or 
aluminum alloy respectively. The scope of the sample in three directions is $(5 \mathrm{~mm} \times 10 \mathrm{~mm} \times 1 \mathrm{~mm}) \sim(50 \mathrm{~mm} \times 50 \mathrm{~mm} \times 1 \mathrm{~mm})$, The mass range of the load is $0.135(\mathrm{~g}) \sim 22.25(\mathrm{~g})$.

\section{Principle of the design}

Rated power of Ac speed motor is $100 \mathrm{~W}$, maximum power output within $30 \mathrm{~min}$ is $110 \mathrm{~W}$. Rated speed is $1500 \mathrm{r} / \mathrm{min}$, maximum speed of revolution is $4000 \mathrm{r} / \mathrm{min}$, minimum rotated speed is $12.7 \mathrm{r} / \mathrm{min}$. Calculation speed is $254 \mathrm{r} / \mathrm{min}$, minimum rotated speed is $6 \mathrm{r} / \mathrm{min}$. Tandem mechanical transmission, $Z=2$. Maximum speed of revolution is $4000 \mathrm{r} / \mathrm{min}$ 。

Range of constant power of the principal axis

$$
R_{n N}=\frac{n_{\max }}{n_{j}}=\frac{4000}{254}=15.7
$$

Range of constant power of the motor

$$
R_{d N}=\frac{n_{d \max }}{n_{d e}}=\frac{4000}{1500}=2.67
$$

Speed range of mechanical transmission

$$
R_{f N}=\frac{R_{n N}}{R_{d N}}=\frac{15.7}{2.67}=5.88
$$

Common ratio of mechanical transmission

$$
\varphi_{f}=\sqrt[Z-1]{R_{f N}}=5.88
$$

Structural gradual

$$
Z=2_{1}
$$

Minimum common ratio of mechanical graded transmission systems

$$
i_{\min }=\frac{n_{j}}{n_{0}}=\frac{254}{1500}=\frac{1}{5.88^{1.0029}}
$$

Minimum reduction ratio of mechanical graded drive system

$$
i_{a 1}=\frac{1}{5.88^{0.6298}}=\frac{1}{3.05}\left[\approx \frac{24}{61}\right]>\frac{1}{4}, i_{0}=\frac{1}{5.88^{0.3732}}=\frac{1}{1.94}\left[\approx \frac{34}{66}\right]>i_{a 1}
$$

Transmission ratio of other transmission pairs

$$
i_{a 2}=i_{a 1} \varphi_{f}=5.88 \times \frac{1}{5.88^{0.6298}}=5.88^{0.3732}=1.94\left[\approx \frac{66}{34}\right]
$$

Minimum rotated speed of a speed regulating motor

$$
n_{d \min }=\frac{n_{\min }}{i_{\min }}=10.2 \times 5.91 \mathrm{r} / \mathrm{min} \approx 60 \mathrm{r} / \mathrm{min}
$$

Power output of the motor at the minimum rotated speed

$$
P_{d \min }=P_{0} \frac{n_{d \min }}{n_{0}}=18.5 \times \frac{60}{1500} \mathrm{~kW}=0.74 \mathrm{~kW}
$$

Reduction ratio of constant ratio transmission( triangular thread,M20 $\times 1.5$ )

$$
n=\left(\frac{3}{1.5} \sim \frac{60}{1.5}\right) r / \min =(2 \sim 40) r / \min
$$

Reduction ratio of constant ratio transmission

$$
i=\frac{n_{j}}{n_{d \max }}=\frac{254}{40}=6.35
$$

The movement diagram of the mechanism is shown in figure 1 . 


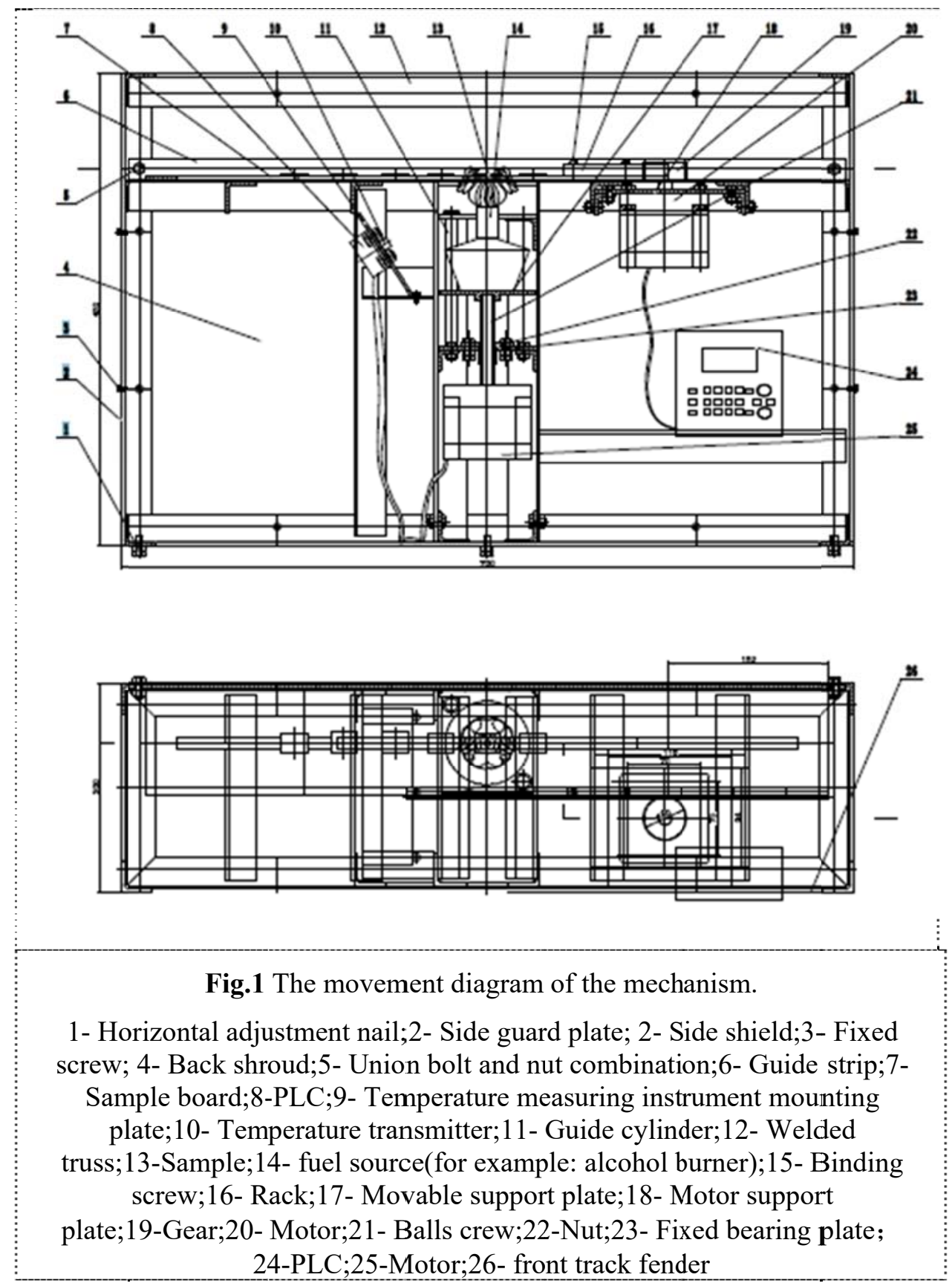

Samples 13 evenly arranged is placed on board7 in test. When the programmable controller24 gives a signal, the gear is driven by stepping motor20 and rack 16 is driven to make a uniform rectilinear motion. Sample layer board7 is linked with rack16 by mechanical separator system in order to do a uniform rectilinear motion. Rectangular holes are processed in board7 to match samples for keeping a touch between samples lower surface and the certain position of the flame.

The flame will gradually weaken with burning, and it makes a effect on concentration of Nano-carbon particles on the lower surface of samples. The temperature of flame in certain position is detected by sensor 10 for keeping it in a suitable range. When it is in a designed section, any single will be not sent; when it goes beyond a reasonable range, an order will be sent by PLC8 to stepping motor25. Ballscrew21 will be driven by it and makes a straight line motion in $\mathrm{Z}$ direction by means of a set of machine combination which consists of nut22, screw21 and other parts in order to keep the temperature.

Nano-carbon particles from the flame of candles, kerosene and soya-bean oil are deposited on the lower surface of samples 13 in this device and the superhydroph0bic template is formed. 


\section{Materials and Methods}

\subsection{Materials}

Glass, stainless steel(304), kerosene, soya-bean oil. Kerosene and soybean oil are placed in the alcohol lamp respectively. Lamp wick is cut neatly before burning.

\subsection{Characterization}

The morphology of the soot particles was characterized by Scanning Electron Microscopy (low voltage LEO 1530 Gemini, Germany, and SU8000,Hitachi, Japan).Samples were washed by high speed centrifuge(TG618, Shanghai,China), and were dried in electrothermal constant-temperature dry box(202-3A,Nanjing,China). Contact angles were tested by Optical Contact Angle Measuring instrument(DSA100,KRUSS,Germany).

\subsection{Methods}

The superhydrophibic deposition layer with Nano-carbon particles was made by Numerical Control Device(Fabricating by Project Group). The ambient temperature stays at the rang 25 degrees Celsius. Outer flame was used when burning. Samples were placed in an airtight plastic box.

\subsection{Rules of samples labelling}

Each sample is marked with three set of numbers. The first set of numbers represents the fuel flame type. For example: 1- Kerosene flame; 2-Candle flame; 3-Soya-bean flame. The second set of numbers represents the base materials. For instance:1-Stainless steel substrate; 2-Glass substrate. The third group represents deposition time (second).

\section{Result and discussion}

Morphology of template and static contact angle were shown in Fig.2 4.

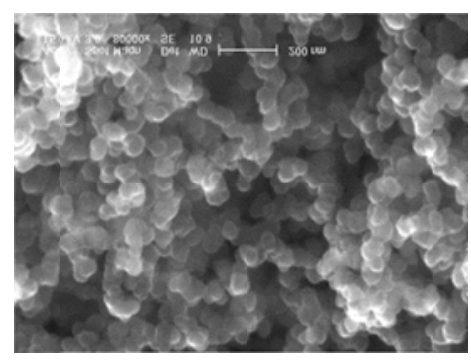

Fig.2(a) 1-1-10 Top view

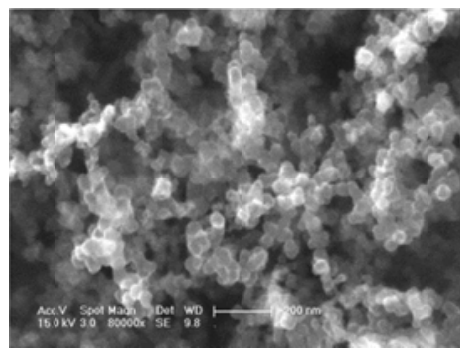

Fig.3 (a) 2-1-30 Top view

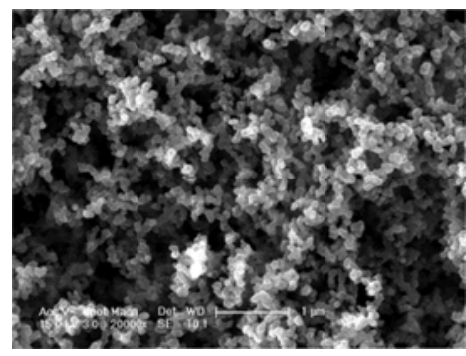

Fig.2 (b) 1-2-10 Top view

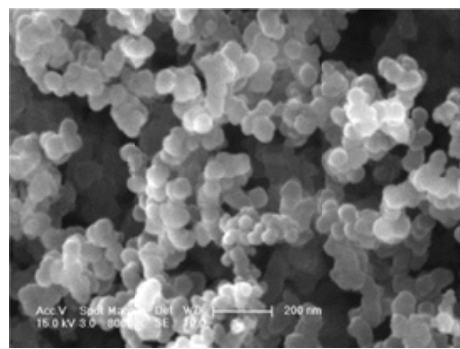

Fig.3 (b) 2-2-30 Top view

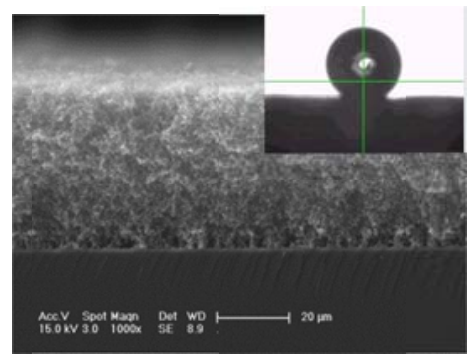

Fig.2 (c) 1-2-10 left view

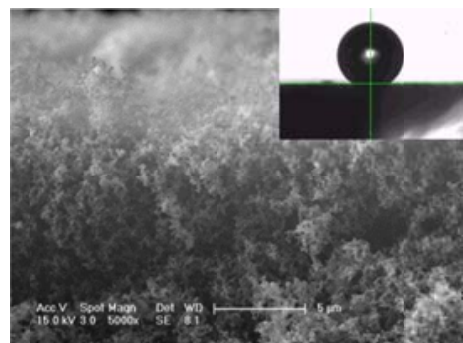

Fig.3(c) 2-2-30 left view 


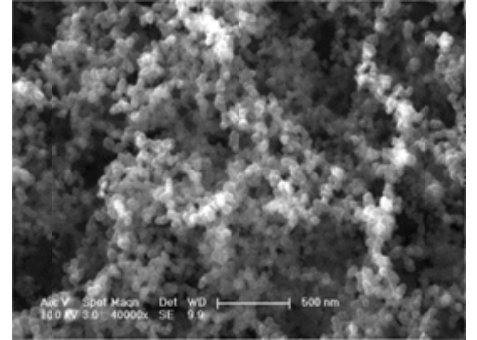

Fig.4 (a) 3-1-30 Top view

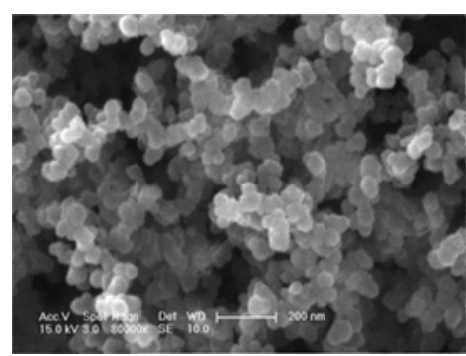

Fig.4 (b) 3-2-30 Top view

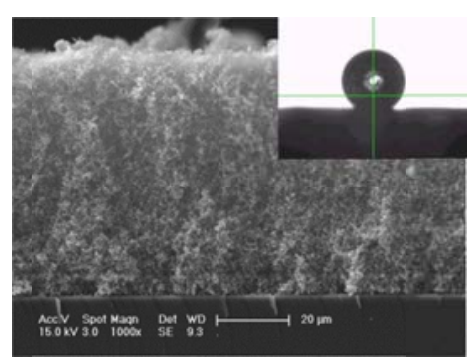

Fig.4(c) 3-2-30 left view

It is shown from morphology of SEM imagine that the diameter of carbon particles on the deposition layer is between 40 nanometer and 50 nanometer. The distribution of particles is random. The carbon particles deposited on the stainless steel substrate have higher densities; The porosity of carbon particles deposited on the base of glass is higher (Shown in Fig.2). With the deposition time increases, the density augment, the porosity reduced, and the thickness of the sediments increases synchronously (Shown in Fig.2 4). The thickness of the sediment is uniformed and is about (50 70) micrometer. It has been indicated that static contact angle is above $150^{\circ}$ (Shown in Fig.2 4c ). Because of the surface energy of glass is lower than that of stainless steel, the contact angle is higher correspondingly.

\section{Conclusion and prospect}

It is indicated in test that the uniform motion in the horizontal direction of the template afforded by the numerical control devices ensures the uniformity of nano-carbon particles on substrates and the static contact angle more than $150^{\circ}$. The superhydrophobic characteristics are presented. It is a foundation for manufacturing the superhydrophobic surface further.

\section{References}

[1].W. Barthlott, C. Neinhuis, Purity of the sacred lotus, or escape from contamination in biological surfaces,Planta. 202 (1997) 1-8.http://dx.doi.org/10.1007/s004250050096

[2]. A. Nakajima, K. Abe, K. Hashimoto, T. Watanabe, Preparation of hard super-hydrophobic films withvisible light transmission, Thin Solid Films. 376 (2000) 140-143.

http://dx.doi.org/10.1016/S0040-6090(00)01417-6

[3]. A. Nakajima, K. Hashimoto, T. Watanabe, K. Takai, G. Yamauchi and A. Fujishima, Transparent

[4]. R. Blossey, Nat. Mater. 2, 301 (2003).

[5].Yan Fang,etc.Superhydrophobic mechanism of butterfly wing non-smooth surface, Science Bulletin,2007.02(52),page354 357. 6. L. C. Gao, T. J. McCarthy, Langmuir 22, 2966 (2006).

[6]. X. Deng et al., Adv. Mater. (Deerfield Beach Fla.) 23,2962 (2011).

[7]. J. Genzer, K. Efimenko, Science 290, 2130 (2000).

[8]. S. H. Kim, S. Y. Lee, S. M. Y5ang, Angew. Chem. Int. Ed.49, 2535 (2010).

[9]. Z. Yoshimitsu, A. Nakajima, T. Watanabe, K. Hashimoto,Langmuir 18, 5818 (2002).

[10]. A. B. D. Cassie, S. Baxter, Trans. Faraday Soc. 40,0546 (1944).

[11]. A. Lafuma, D. Quéré, Nat. Mater. 2, 457 (2003).

[12]. Q. Xie et al., Adv. Mater. (Deerfield Beach Fla.) 16, 302(2004).

[13]. Xu Deng, Lena Mammen, Hans-Jürgen Butt, Doris Vollmer Candle Soot as a Template for a

Transparent Robust Superamphiphobic Coating, SCIENCE VOL 3356 JANUARY 2012,67 69. 